[0212-7199 (2008) 25: 6; pp 279-283] ANALES DE MEDICINA INTERNA Copyright (C) 2008 ARAN EDICIONES, S.L.

AN. MED. InTERnA (Madrid) Vol. 25, N. ${ }^{\circ} 6$, pp. 279-283, 2008

\title{
Leishmaniasis visceral y asma bronquial: influencia del tratamiento esteroideo en el desarrollo del síndrome de activación macrofágica e insuficiencia suprarrenal relativa
}

\author{
M. A. RUIZ GINÉS ${ }^{1}$, J. A. RUIZ GINÉS ${ }^{2}$, J. L. MENÉNDEZ GÓMEZ ${ }^{3}$, A. M. PÉREZ \\ COGOLLUDO $^{3}$, S. DOMÍNGUEZ MARTÍN ${ }^{3}$, E. FERNÁNDEZ RODRÍGUEZ ${ }^{1}$
}

\begin{abstract}
${ }^{\text {}}$ Servicio de Análisis Clínicos y Bioquímica y ${ }^{2}$ Servicio de Urgencias. Hospital Virgen de la Salud. Toledo. CEDT de Illescas. Toledo. ${ }^{I}$ Servicio de Neurología. Hospital Universitario de La Princesa. Madrid
\end{abstract}

VISCERAL LEISHMANIASIS AND BRONCHIAL ASTHMA: INFLUENCE OF STEROID THERAPY IN THE DEVELOPMENT OF THE MACROPHAGE ACTIVATION SYNDROME AND RELATIVE ADRENAL INSUFFICIENCY

\section{RESUMEN}

El riesgo de padecer infecciones oportunistas en los pacientes inmunodeprimidos es un hecho perfectamente establecido. Una situación infrecuente la constituyen las broncopatías, patologías con un elevada prevalencia entre la población general, que requieren habitualmente, entre otros, tratamiento esteroideo. La inmunodepresión confiere a la evolución clínica de las infecciones, una inadecuada respuesta al estrés físico, debido a la inhibición del eje hipotálamo-hipofisario, pudiendo en casos particularmente graves, desarrollar el denominado Síndrome de Activación Macrofágica, síndrome grave e infrecuente, que ensombrece el pronóstico clínico en estos pacientes.

Ante un síndrome febril de origen incierto en un paciente en tratamiento inmunosupresor, aunque sea a dosis bajas, es necesario realizar un exhaustivo diagnóstico diferencial, debiendo considerar, entre ellos, la infección por Leishmania, una parasitosis cuya incidencia se está incrementando notablemente en los últimos años entre la población inmunodeprimida.

Se presenta el caso clínico de un paciente de 63 años de edad, inmunocomprometido a consecuencia de terapia crónica esteroidea secundaria a broncopatía asmática, que experimenta una forma infrecuente de leishmaniasis visceral en nuestro medio, consistente en fracaso multiorgánico a raíz del desarrollo de un Síndrome de Activación Macrofágica.

PALABRAS CLAVE: Asma. Corticoides. Inmunodepresión. Leishmaniasis visceral. Síndrome de Activación Macrofágica. Sepsis. Insuficiencia suprarrenal.

\section{ABSTRACT}

The risk of suffering opportunistics infections in the immunoincompetent patient is a fact perfectly established. An uncommon situation constitutes the bronchopaties, pathologies with a high prevalence among the general population that they require habitually, among other, steroid treatment. The immunosupression confers to the clinical evolution of the infections, as a consequence of the inadequate response to the physical stress, due to the inhibition of the hypothalamus-hypophysis axis being able to in particularly serious cases, to develop the denominated macrophage activation syndrome, a serious and uncommon syndrome that darkens the clinical prognosis in these patients.

In presence of a feverish syndrome of uncertain origin in a patient in immunosuppressor treatment, although it is to low dose, it is necessary to carry out a exhaustive differential diagnosis, should consider, among them, the infection for Leishmania, a parasitosis whose incidence is increasing notably in the last years in the immunosuppressed population.

We present the clinical case of a 63 year-old patient, immunoincompetent as a consequence of secondary chronic steroid therapy to asthmatic bronchopaty that experiences an uncommon form of visceral leishmaniasis in our area, consistent in multiorganic failure in the context of the development of a macrophage activation syndrome.

KEY WORDS: Asthma. Corticoids. Immunosuppression. Visceral Leishmaniasis. Macrophage activation syndrome. Sepsis. Adrenal insufficiency.

Ruiz Ginéz MA, Ruiz Ginés JA, Menéndez Gómez JL, Pérez Cogolludo AM, Domínguez Martín S, Fernández Rodríguez. E. Leishmaniasis visceral y asma bronquial: influencia del tratamiento esteroideo en el desarrollo del síndrome de activación macrofágica e insuficiencia suprarrenal relativa. An Med Interna (Madrid) 2008; 25: 279-283.

\section{INTRODUCCIÓN}

El síndrome febril de origen desconocido en el paciente inmunodeprimido es un cuadro frecuente en los Servicios de Urgencias de todo el mundo (1-4). La etiología más frecuente es la infecciosa, existiendo otras causas de gran prevalencia, particularmente de tipo neoplásico, metabólico, inmunológico, etc. Sin embargo, a pesar de todo, hay causas poco frecuentes y que en muchas ocasiones pasan desapercibidas en las fases iniciales de la anamnesis y exploración física del paciente. Es el caso de la leishmaniasis (5). Se trata de una parasitosis, producida por un grupo de protozoos, cuyo reservorio son cánidos y roedores y cuya transmisión se produce al hombre, en nuestro medio, mediante mosquitos del género Phlebotomus $(6,7)$. Existen varias especies patógenas para el hombre, con diferencias en cuanto a la clínica y la distribución geográfica. Leishmania infantum es la principal responsable de la clínica en nuestro medio, de tipo viscerotropa, pro-

Trabajo aceptado: 14 de enero de 2008 
duciendo el denominado Kala-azar, leishmaniasis visceral o fiebre dum-dum. Su incidencia se ha incrementado notablemente en los últimos años, particularmente entre la población inmunodeprimida. En estos sujetos, la funcionalidad del sistema inmune está notablemente afectada, lo que se traduce en una activación de la infección latente o en una respuesta insuficiente a la infección activa, con un curso clínico habitualmente agresivo, debido, fundamentalmente, al desarrollo del síndrome de activación macrofágica (SAM) (21-3), un síndrome grave e infrecuente, cuya base radica en la alteración de la inmunorregulación, traduciéndose en una respuesta masiva, inespecífica y desproporcionada, con las consiguientes repercusiones sistémicas de carácter multiorgánico.

Presentamos el caso de un paciente que ingresó en el Servicio de Urgencias de nuestro hospital, afectado de un síndrome febril, fracaso multiorgánico e inestabilidad hemodinámica asociada, sin un foco desencadenante conocido inicialmente, pero que a partir de exhaustivos y sistemáticos exámenes complementarios, pudo establecerse como agente causal, un protozoo del género Leishmania.

\section{CASO APORTADO}

Paciente de 63 años de edad, sin alergias medicamentosas conocidas. Diabetes mellitus tipo 2 diagnosticada hace un mes, en tratamiento con antidiabéticos orales. Exfumador. Sobrepeso (IMC:27). Asma bronquial de diez años de evolución, en tratamiento combinado con un anticolinérgico de larga acción (bromuro de tiotropio), un antagonista leucotriénico (Montelukast), corticoides orales e inhalados (deflazacort $6 \mathrm{mg}$ /día y fluticasona en dosis de 400 microgramos/día, respectivamente). Hernia hiatal. Estenosis aórtica leve degenerativa. Hiperplasia benigna de próstata. No presentó viajes al extranjero, consumo de lácteos sin pasteurizar ni caza. Dueño de un perro, con todos los controles sanitarios reglamentarios en orden.

Acude al Servicio de Urgencias por clínica de quince días de evolución consistente en fiebre de predominio vespertino, malestar general, sudoración profusa, astenia, pérdida de peso, vómitos, dolor epigástrico y somnolencia creciente. En las últimas 24 horas, la fiebre se ha tornado continua, con escalofríos y tiritona asociados. No presenta datos adicionales en relación con clínica de infección a nivel del sistema nervioso central, vías respiratorias altas, vías respiratorias bajas (tos o incremento de la expectoración), abdomen (dolor abdominal o síndrome diarreíco), sistema urinario (disuria, polaquiuria, tenesmo o urgencia miccional). Destaca un episodio de odontalgia diez días antes en relación con absceso peridental, resuelto con antibioterapia oral (amoxicilina-clavulánico). Ante el progresivo deterioro del estado general del paciente, con hipotensión arterial y tricitopenia asociadas, se decide su ingreso hospitalario en la Unidad de Cuidados Intensivos, con el juicio clínico de shock séptico en relación con síndrome febril de etiología desconocida.

Exploración física Destaca, al ingreso, una tensión arterial de $89 / 57 \mathrm{~mm} \mathrm{Hg}$, con una temperatura timpánica de $39,3^{\circ} \mathrm{C}$. El paciente se mostraba consciente, orientado globalmente, con deshidratación cutáneo-mucosa y palidez cutánea, aunque con mucosas normocoloreadas. Destaca un pequeño hematoma en la cara interna del brazo izquierdo. No se objetivan lesiones cutáneas infeccioso-inflamatorias. En el estudio de la cabeza y cuello, las carótidas eran rítmicas y simétricas, sin aumento de la presión venosa yugular, adenopatías, ni puntos dolorosos a la palpación. No se objetivó bocio. A nivel torácico, la auscultación cardíaca era rítmica, revelando taquicardia $(120 \mathrm{lpm})$, sin soplos ni extratonos audibles. Taquipneíco en reposo (24 rpm), hipoventilando en ambas bases pulmonares. El abdomen presentaba ruidos hidroaéreos, siendo blando y depresible, aunque doloroso a la palpación a nivel de epigastrio e hipocondrio derecho, objetivándose hepatoesplenomegalia. No presentaba signos de irritación peritoneal. En miembros inferiores se observaban ligeros edemas en tercio distal de ambas piernas. La exploración neurológica reveló unas funciones superiores conservadas, sin alteración en pares craneales, sistema motor, sensitivo ni cerebelo. Los signos orientadores de meningismo (Kernig, Brudzinski y Lasègue), resultaron negativos.

- Pruebas complementarias:

- Hemograma (resumen de los datos más relevantes): Respecto a la serie roja, destacaba una cifra de hemoglobina de $10,1 \mathrm{~g} / \mathrm{dl}$, con un VCM de 78,6 fl. Se descartó sangrado activo y el perfil férrico se encontraba dentro de límites normales, a excepción de una hiperferritinemia de $837,8 \mathrm{ng} / \mathrm{ml}$. La serie blanca reveló una leucopenia moderada $(2.250 \mathrm{cel} / \mu \mathrm{l})$. La cifra de plaquetas mostró valores de 65.200 cel/ $\mu$ l. En extensión de sangre periférica con tinción de May-Grünwald-Giemsa se observó presencia de linfocitos activados y neutrófilos vacuolados. Los estudios analíticos previos, realizados un año antes, eran absolutamente normales.

- Bioquímica: En las determinaciones realizadas en los días previos al ingreso, destaca una glucemia media en torno a los 140 $\mathrm{mg} / \mathrm{dl}$, pero que al ingreso era de $424 \mathrm{mg} / \mathrm{dl}$. Asimismo, presentaba una hiponatremia moderada $(126 \mathrm{mEq} / \mathrm{l})$, con una leve hipocloremia $(93,1 \mathrm{mEq} / \mathrm{l})$. Osmolaridad calculada de $281,4 \mathrm{mOsmol} / \mathrm{l}$. La cifra de Potasio estaba dentro de los límites normales $(4,31 \mathrm{mEq} / \mathrm{l})$. La función renal estaba conservada. Presentaba datos analíticos de desnutrición, con proteínas totales de 4,34 g/dl y una Albúmina de 2,6 g/dl.

Respecto a los distintos perfiles, el hepático mostraba una hipertransaminasemia moderada, de perfil tanto citolítico como colestásico (GOT/AST: $128 \mathrm{mU} / \mathrm{ml}$; GPT/ALT: $82 \mathrm{mU} / \mathrm{ml}$; G-GT: $214 \mathrm{mU} / \mathrm{ml}$, FA: $155 \mathrm{mU} / \mathrm{ml}$ ), una LDH de $643 \mathrm{mU} / \mathrm{ml}$ y una Aldolasa de 16 $\mathrm{mU} / \mathrm{ml}$. A nivel lipídico, destaca una importante hipertrigliceridemia de $400 \mathrm{mg} / \mathrm{dl}$. Respecto al perfil tiroideo y vitamínico, no se observaron anomalías reseñables. El estudio de coagulación reveló una coagulopatía incipiente, con alteración tanto de la vía extrínseca (INR: 1,4; TP: 68\%), como de la intrínseca (TTPA: 38,1"/30"), con una cifra de Fibrinógeno en el límite alto de la normalidad (547 mg/dl). La PCR estaba muy elevada (>90 mg/l). Sin embargo, la VSG no era significativa (12 y $37 \mathrm{~mm}$ en la $1^{\mathrm{a}}$ y $2^{\mathrm{a}}$ hora, respectivamente).

En cuanto a la gasometría arterial practicada, presentaba una alcalosis respiratoria $\left(\mathrm{PH}: 7,55 ; \mathrm{PCO}_{2}: 26 \mathrm{mmHg} ; \mathrm{HCO}_{3}^{-}: 22,7\right.$ $\mathrm{mmol} / \mathrm{L}$ ), con una cifra de $\mathrm{PO}_{2}$ de $63 \mathrm{mmHg}$ mostrando hipoxemia y muy cerca de la insuficiencia respiratoria parcial. La saturación de oxígeno era del $95 \%$. Los niveles de ácido láctico eran adecuados.

En el estudio de orina, se objetivó un incremento de los cuerpos cetónicos, sin ningún otro dato relevante.

En el proteinograma no se observaron bandas de tipo monoclonal.

Respecto a las serologías practicadas, las correspondientes a VHA, VHB, VHC, VIH, virus Epstein Barr, rubéola, varicela, CMV, Brucella, sífilis, Legionella, Mycoplasma penumoniae, Toxoplasma e hidatidosis, resultaron negativas, al igual que el antígeno de Leishmania en orina. Sin embargo, el estudio IgG-Leishmania (IFI) resultó positivo a una dilución de 1/80. Estudio para micobacterias negativo. Asimismo, el estudio de parásitos intestinales resultó negativo.

Las pruebas de imagen iniciales mostraron un índice cardiotorácico dentro de la normalidad, con hilios congestivos, presencia de múltiples adenopatías, de pequeño tamaño, paratraqueales, con mínimo derrame pleural de predominio izquierdo, sin datos de condensación parenquimatosa. La placa abdominal no mostró alteraciones.

En vista de estos resultados se decide practicar ecografía abdominal, que reveló esteatosis hepática, colelitiasis y una gran esplenomegalia (177 mm de diámetro longitudinal), con múltiples lesiones nodulares hiperecogénicas, mal delimitadas, difusas, así como un angiomiolipoma de $4 \mathrm{~mm}$ en corteza renal izquierda. Todos estos hallazgos se confirmaron en el estudio mediante TAC abdominal.

Se practicaron hemocultivos, con el fin de descartar probable causa infecciosa con diseminación metastásica, que revelaron Staphylococcus epidermidis (contaminante) en 1 de 6 determinaciones. El estudio ecocardiográfico descartó endocarditis. 
En la biopsia de médula ósea, se observa celularidad global incrementada, megacariocitos en proporción normal, una serie granulocítica bien representada, con una serie eritropoyética hiperplasiada, con rasgos diseritropoyéticos incrementados. Sideremia macrofágica exacerbada. Sideroblastos negativos y macrófagos con imágenes de fagocitosis. Asimismo, imágenes extracelulares sugerentes de infección por Leishmania.

En vista de la inestabilidad clínica del paciente, se decide su ingreso en la Unidad de Cuidados Intensivos, con el diagnóstico de shock séptico secundario a leishmaniasis visceral o Kala-Azar.

\section{DISCUSIÓN}

Analizando los resultados de las pruebas complementarias de una forma sistemática, podemos comenzar justificando la tricitopenia en relación con la esplenomegalia del paciente y una destrucción celular acelerada. De igual forma, el cuadro metabólico consistente en desnutrición y descompensación hiperglucémica tendente a la hiperosmolaridad, es justificable en relación con una respuesta vegetativa hipercatabólica sostenida. La hiponatremia observada está en relación con una depleción volumétrica, y no con una pesudohiponatremia lipídica, siendo la causa última más plausible el hipoaldosteronismo. Para confirmarlo, se procedió a realizar en nuestro paciente, determinación de cortisol basal siguiendo las pautas de Marik y Zaloga (18) que fijan en 23,7 $\mu \mathrm{g} / \mathrm{dl}$ el valor límite del cortisol para discriminar entre normalidad e insuficiencia corticosuprarrenal, obteniendo unas cifras de cortisol basal de $21,3 \mu \mathrm{g} / \mathrm{dl}$ y cortisol $60 \mathrm{~min}$ tras estimulación con ACTH de $33,6 \mu \mathrm{g} / \mathrm{dl}$, lo que orienta hacia una insuficiencia corticosuprarrenal relativa (sin deterioro estructural del eje hipotálamohipófisis-suprarrenal) en un paciente crítico y en tratamiento crónico con altas dosis de esteroides (orales e inhalados). Estos autores concluyen que todo paciente inmerso en un cuadro clínico compatible con shock séptico debe recibir tratamiento con Hidrocortisona con mantenimiento posterior si la cifra del mismo es inferior a $25 \mu \mathrm{g} / \mathrm{dl}$. Se instauró, por ello, tratamiento sustitutivo con Hidrocortisona (100 mg/8 horas). También mantienen la administración de Hidrocortisona en los pacientes que, a pesar de tener valores de Cortisol elevados, presenten una buena respuesta hemodinámica a dicho tratamiento, ya que este tratamiento supone muy poco riesgo adicional (20). Se consideró esta opción por la notable mejoría hemodinámica que experimentó el paciente, aunque no se hubiera podido confirmar la presencia de una insuficiencia suprarrenal según los autores Cooper y Stewart (19) (Fig. 1). En cuanto a la hepatopatía, en ausencia de ictericia, una vez descartada la hepatitis (A, B y C), colangitis, ingesta de agentes hepatotóxicos y la cardiopatía isquémica (troponina I: 0,019 ng/ml, CK: $37 \mathrm{mU} / \mathrm{ml}$ ), resta el origen séptico infeccioso. Asimismo, la coagulopatía que presenta el paciente se explica por un déficit de factores dependientes de la vitamina $\mathrm{K}$ (déficit parcial de los factores II, VII y el X) y del Factor XII de la vía intrínseca, así como un déficit en la actividad de la antitrombina III (55\%). Destaca una falta de elevación de la VSG, justificable por la consecuente activación de la cascada coagulatoria, no siendo un parámetro útil en la monitorización de esta patología.

Un elemento esencial en nuestro caso es la coexistencia de fiebre, tricitopenia, hipertrigliceridemia, hiperferritinemia, incremento de $\mathrm{LDH}$, presencia de hemofagocitosis tanto en médula como en sangre periférica y esplenomegalia, ya que

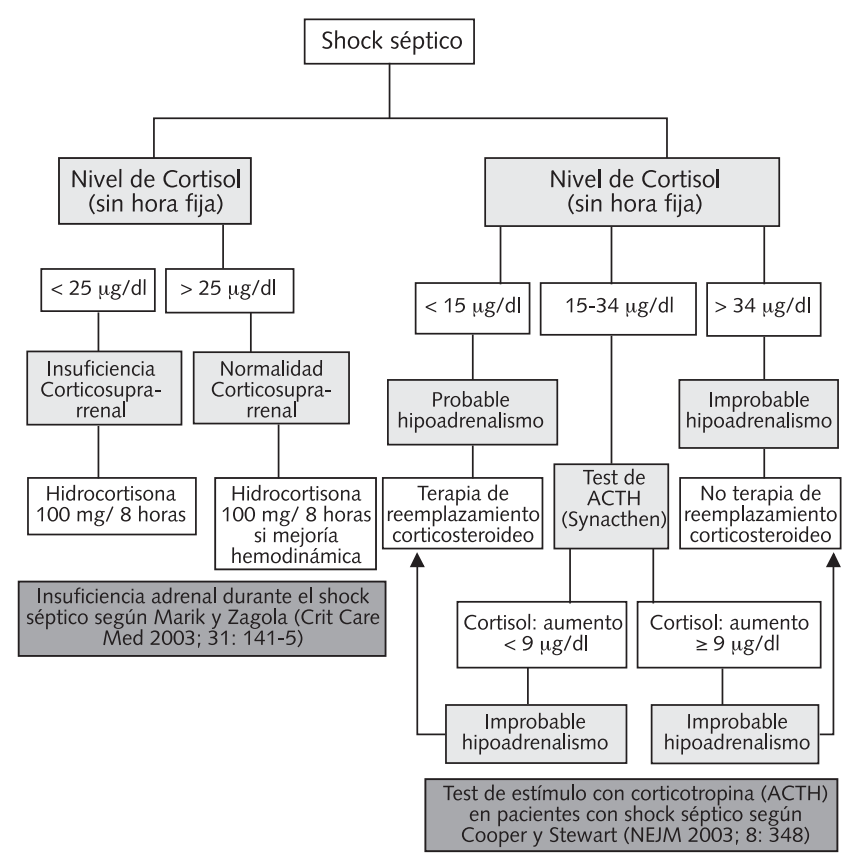

Fig. 1. Estudios para el diagnóstico de insuficiencia suprarrenal.

constituyen los criterios diagnósticos del SAM (descrito por Stephan et al, 1993) (Tabla I). Se trata de un síndrome infrecuente y muy grave (21-24), que afecta predominantemente a niños, ancianos, pacientes debilitados por enfermedades crónicas, así como en inmunodeprimidos. La fisiopatología del mismo se basa en una alteración de la inmunorregulación, lo que genera una respuesta inmunológica exagerada, con una sobreproducción de citoquinas (interferón y factor estimulante de colonias granulocítico-macrofágica particularmente). El resultado es una alteración de la capacidad citotóxica de las células $\mathrm{NK}$ y de los linfocitos $\mathrm{T}$, junto con una activación macrofágica, con infiltración tisular y producción de nuevas citoquinas (TNF, IL-1 e IL-6), fundamentales en la progresión de la lesión tisular y el inicio de los síntomas. Entre las posi-

\section{TABLA I}

\section{CRITERIOS DIAGNÓSTICOS DEL SÍNDROME DE ACTIVACIÓN MACROFÁGICA}

\section{Clinicos}

Fiebre: picos de $38,5^{\circ} \mathrm{C}$ o más durante al menos siete días

Esplenomegalia de al menos $3 \mathrm{~cm}$

\section{Laboratorio}

Citopenia de dos o más líneas celulares:

Anemia: $\mathrm{Hb}<9 \mathrm{mg} / \mathrm{dl}$

Trombocitopenia: $<100.000$ plaquetas $/ \mathrm{mm}^{3}$

Neutropenia: $<1.000$ neutrófilos $/ \mathrm{mm}^{3}$

Hipertrigliceridemia $>180 \mathrm{mg} / \mathrm{dl}$ ( $0>3$ desviaciones estándar para

cada edad) o hipofibrinogenemia $<150 \mathrm{mg} / \mathrm{dl}$

Histopatológicos

Hemofagocitosis en médula ósea, bazo o ganglio linfático, sin evidencia de malignidad 
bles causas productoras de este síndrome destacan procesos infecciosos, neoplásicos, colagenopatías, inmunodeficiencias, fenómenos postvacunales, sarcoidosis, terapia inmunosupresora, etc. (Tabla II y Fig. 2). Dentro de las causas infecciosas parasitarias encontramos a la producida por Leishmania, ocupando un puesto relevante.

Se puede catalogar, por tanto, el cuadro clínico como de leishmaniasis visceral o Kala-Azar en paciente inmunodeprimido, con respuesta minimizada ante una situación de estrés físico en relación con insuficiencia suprarrenal yatrogénica subyacente, junto con un síndrome de activación macrofágica asociado. Este tipo de parasitosis es producida por protozoos del género Leishmania. La especie es dependiente del ámbito geográfico, de tal forma que en los países mediterráneos, es L. infantum la forma predominante. El número de casos anuales registrados, en nuestro medio, se sitúa, aproximadamente, en $100(0,81 / 100.000$ habitantes) (26), aunque con una incidencia creciente. El vector transmisor, es un mosquito del género Phlebotomus. En cuanto al reservorio, se establecen dos grandes grupos epidemiológicos,

\section{TABLA I}

\section{CAUSAS DEL SÍNDROME DE ACTIVACIÓN MACROFÁGICA}

-Infecciones

- Virales: virus Epstein Barr (VEB), Citomegalovirus, Herpes simple, Varicela Zoster, Rubeola, VIH, Adenovirus, Arbovirus, Parvovirus, hepatitis A y C, Influenzae, Coxsackie

- Bacterias: Micobacterias, estafilococos, Streptococcus pneumoniae, Legionella, Brucella, Haemophilus, Serratia

- Hongos: Candida, histoplasma, cryptococcus

- Parásitos: Leishmania, Toxoplasma, Chlamydia

-Colagenopatías

- LES (Lupus eritematoso sistémico), ARJ (Artritis reumatoide juvenil), enfermedad de Kawasaki

-Neoplasias

- Carcinomas, Mieloma múltiple, Linfomas, Leucemias, Síndromes mielodisplásicos

-Inmunodeficiencias primarias

- Síndrome de Chediak-Higashi, Síndrome de Griscelli

-Otras

- Fármacos (Fenitoína, inmunosupresores como Metotrexato), vacunas, Sarcoidosis, hiperalimentación parenteral con lípidos

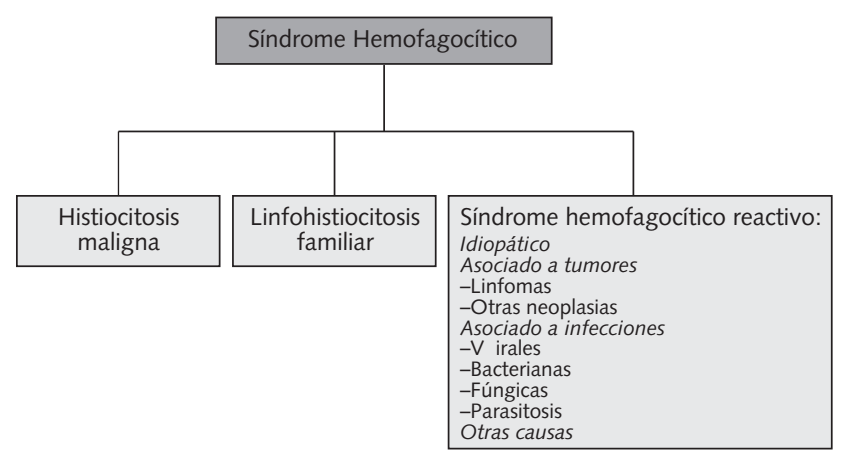

Fig. 2. Clasificación del síndrome de activación macrofágico. el antroponótico, donde el ciclo comienza y termina en el humano y el zoonótico, donde el ciclo se inicia en el vertebrado no humano (el reservorio principal es el perro, con una prevalencia de hasta el $15 \%$, según las áreas) y se cierra en el humano. El ciclo biológico, en el reservorio final, concluye con la inoculación de las formas flageladas, los promastigotes, que se acumulan a nivel intracelular en el sistema mononuclear-fagocítico (macrófago), como estrategia para escapar a la respuesta inmune inespecífica del huésped vertebrado, quedando englobados en el interior de vacuolas, donde adquieren la forma definitiva aflagelada o amastigotes. Se trata de una parasitosis emergente en nuestro medio, en los últimos años, particularmente entre sujetos inmunodeprimidos (VIH, fármacos, pacientes trasplantados y enfermos oncológicos).

Los principales órganos diana son hígado, bazo y médula ósea. Cursa de forma insidiosa, con fiebre, astenia y pérdida de peso (8-10). Puede aparecer en el punto de picadura, una lesión cutánea, que simule un epitelioma. Posteriormente, la fiebre se acentúa, apareciendo molestias gastrointestinales (estreñimiento o diarrea y vómitos). La ascitis puede estar presente en el 5-10\% de los casos. Aparece palidez y sequedad de piel. Se observa hepatomegalia homogénea $(8,11)$ de superficie lisa y consistencia blanda, así como esplenomegalia importante y dolorosa. Pueden observarse petequias, equimosis, epistaxis y edemas. Existen adenopatías cervicales, fundamentalmente. La muerte suele acontecer por infección bacteriana secundaria, hemorragia o anemia.

En cuanto al diagnóstico, podemos emplear métodos directos, basados en la visualización de los amastigotes intramacrófágicos, en la biopsia de médula ósea, mediante el empleo de tinciones a base de Giemsa, así como su cultivo en medios específicos, como el de Novy-Nicolle-McNeal (o medio NNN). En cuanto a los métodos indirectos, de base inmunológica, las técnicas de inmunofluorescencia permiten detectar anticuerpos IgG frente al parásito.

El tratamiento de elección de esta enfermedad son los antimoniales a dosis de $20 \mathrm{mg} / \mathrm{kg} /$ día durante 3-4 semanas. Existe otra alternativa para aquellos pacientes que no responden, recidivan o presentan importantes reacciones secundarias a los mismos, como es la Pentamidina a dosis de $4 \mathrm{mg} / \mathrm{kg} /$ día durante 3-4 semanas. En este paciente, se empleó la Anfotericina B liposomal a dosis de $5 \mathrm{mg} / \mathrm{kg} /$ día durante cinco días y, posteriormente una dosis semanal durante cuatro semanas, por tratarse de un paciente inmunodeprimido y poseer menor nefrotoxicidad y mayor capacidad de concentración tisular $(10,16,17,26)$. El paciente presentó una buena respuesta al tratamiento, encontrándose asintomático hasta la fecha.

En conclusión, podemos afirmar:

1. Si bien está perfectamente establecido el riesgo de padecer infecciones oportunistas en los pacientes inmunodeprimidos, ya sean enfermos afectados por el virus de la inmunodeficiencia humana (VIH), oncológicos, trasplantados o en tratamiento farmacológico, una situación infrecuente la constituyen las broncopatías (asma, EPOC, etc...), patologías con elevada prevalencia entre la población general, que requieren tratamiento crónico esteroideo, por vía inhalada u oral. Actualmente sólo existe descrito un caso de Leishmaniasis en este grupo de pacientes (25).

2. Los pacientes inmunodeprimidos muestran una serie de características singulares en la evolución clínica de las infecciones, que le confieren un peor pronóstico, dada la tendencia a desarrollar el SAM y a la falta de una respuesta adecuada ante situaciones de estrés físico, debido a la inhibición del eje hipotálamo-hipofisario. 
3. Por ello, es esencial, en todo paciente en tratamiento inmunosupresor, aunque sea a dosis bajas, considerar dentro del diagnóstico diferencial del síndrome febril de origen incierto, la infección por Leishmania, dado el alto riesgo de infecciones oportunistas subyacente que presentan este grupo de pacientes, como forma de poder iniciar el tratamiento oportuno de la forma más precoz posible.

\section{Bibliografía}

1. Amin K, Kauffman CA. Fever of unknown origin. A strategic approach to this diagnostic dilemma. Postgrad Med 2003; 114: 69-75.

2. Arnow PM, Flaherty JP. Fever of unknown origin. Lancet 1997; 350: 575-80.

3. Carpenter CCJ. Fever of undetermined origin. In: Barondess JA, Carpenter CCJ (eds). Differential Diagnosis. Filadelfia, Lea and Febiger 1994; 503-56.

4. Laso FJ. Fiebre. En: Diagnóstico diferencial en Medicina Interna. Elsevier 2006; 3-10.

5. Desjeux P: The increase in risk factors for leishmaniasis worldwide. Trans R Soc Trop Med Hyg 2001; 95: 239-43.

6. Bryceson ADM. Leishmaniasis. In: Manson ed. Manson's Tropical Diseases. $20^{\text {th }}$ ed. WB Saunders 1996; 1213-45.

7. Fritsche TR, Selvarangan R. Medical Parasitology. In: Henry's Clinical Daignosis and Management by Laboratory Methods. $21^{\text {th }}$ ed. Saunders Elsevier 2007; 1135-36.

8. Prasad LS. Kala azar. Indian J Pediatr 1999; 66: 539-46.

9. Herwaldt BL. Leishmaniasis. Lancet 1999; 354: 1191-9.

10. De Diego Lorenzo A, et al. Leishmaniasis in a patient with liver cirrhosis. An Med Interna (Madrid) 2003; 20: 145-7.

11. Buyukasik Y, Ileri NS, Haznedaroglu IC, et al. Fever, hepatosplenomegaly and pancytopenia in a patient living in the Mediterranean region. Postgrad Med J 1998; 74: 237-9.

12. Guardia J, Fraiha HN, Miles MA. Leishmaniasis y tripanosomiasis. EN: Verger Garau G. Enfermedades Infecciosas. Ed. Doyma SA, Barcelona 1988: 410-4.

13. Medina Pérez M, Jiménez González F, Marín Martín J. Leishmaniasis en el bloque celular del aspirado de médula ósea. An Med Interna (Madrid) 2000; 17: 72-3.

14. Khaldi F, Bennacem B, Othaman HB, et al. Les formes severes d'attein-
4. Probablemente, la mayoría de las linfohistiocitosis hemofagocíticas (Síndrome de Activación Macrofágica) vistas en la práctica clínica estén relacionadas más con agentes infecciosos que con enfermedades reumáticas, siendo necesario una estrecha colaboración con el Médico Analista Clínico y el Microbiólogo a fin de aclarar la posible causa infecciosa subyacente, siendo la mayoría tratables y con buen pronóstico. te hepatique au cours de la leichmaniose viscerale. A propos de 7 cas. Arch Fr Pediatr 1990; 47: 257-60.

15. Cañería MV, et al. Atypical presentation of Visceral Leishmaniasis. JAPI 2005; 53: 573-5.

16. Singh UK. Diagnosis and management of kala azar. Indian Peditr 1999; 36: 231-6.

17. Gangneux JP. Treatment of visceral leishmaniasis: recent modalities. Presse Med 1999; 28: 2057-66.

18. Marik PE, Zaloga GP. Adrenal insufficiency during septic shock. Crit Care Med 2003; 31: 141-5.

19. Cooper MS, Stewart PM. Corticosteroid insufficiency in acutely ill patients. N Engl J Med 2003; 348: 727-34.

20. Lopez-Martínez J, Suárez-Saiz J. Insuficiencia corticosuprarrenal en el paciente crítico. Med Intensiva 2004; 28: 208-10.

21. Kouris E, Giansante E. Síndrome de activación macrofágica. Med Cutan Iber Lat Am 2006; 34: 145-54.

22. Sastre Urgellés A. Síndromes hemofagocíticos: pensar en ellos...porque existen. An Esp Pediatr 2002; 56: 95-8.

23. Artigues Barceló A, Ferragut Reus M, Sánchez C, Amengual I, Matanza I, Sanz Parras MS. Síndrome hemofagocítico y linfoma cutáneo de células T. An Med Interna (Madrid) 2004; 21: 131-4.

24. Grande Tejada AM, Cardesa García JJ, Vagace Valero JM, García Domínguez M, Bejarano Ramírez N. Leishmaniasis visceral, a propósito de tres casos. Vox Paeddiatrica 2004; 12 (1): 34-8.

25. Pittalis S, Nicastri E, Spinazzola F, Ghirga P, De Marco M, Paglia MG, et al. BMC Infectious Diseases 2006; 6: 177.

26. Figueras Nadal MC, García de Miguel MJ, Asensi Botet F, Velasco Bernardo R, Canals Baeza A, et al. Tratamiento de corta duración de la leishmaniasis visceral con anfotericina B liposómica en pacientes inmunocompetentes. An Pediatr (Barc) 2003; 59: 535-40. 